\title{
Renin-Angiotensin System Inhibition in Cardiovascular Patients at the Time of COVID19: Much Ado for Nothing? A Statement of Activity from the Directors of the Board and the Scientific Directors of the Italian Society of Hypertension
}

\author{
Guido laccarino $^{1}$ (1) - Claudio Borghi ${ }^{2}$ (i) - Arrigo F. G. Cicero ${ }^{2}$ (D) - Claudio Ferri ${ }^{3}$. Pietro Minuz ${ }^{4}$.

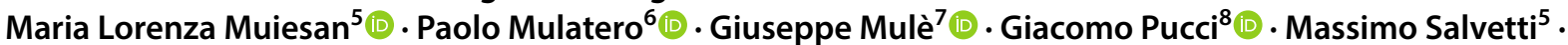 \\ Carmine Savoia ${ }^{9} \cdot$ Leonardo Alberto Sechi $^{10} \cdot$ Massimo Volpe $^{11,12}$ (1) $\cdot$ Guido Grassi $^{13}$ (b)
}

Received: 26 March 2020 / Accepted: 2 April 2020 / Published online: 7 April 2020

(c) The Author(s) 2020

\begin{abstract}
Cardiovascular diseases, in particular hypertension, as well as the cardiovascular treatment with Renin-Angiotensin System inhibitors such as Angiotensin Converting Enzyme (ACE) inhibitors and Angiotensin Receptor Blockers (ARBs), are claimed once again as mechanisms of Severe Acute Respiratory Syndrome (SARS) during the COVID-19 outbreak due to Cov-2 epidemics. In vitro studies are available to support the eventual role of ACE inhibitors and ARBs in both the promotion and antagonism of the disease. The available literature, indeed, presents contrasting results, all concentrated in experimental models. Evidence in humans is lacking that those mechanisms are actually occurring in the present COVID-19 outbreak. Here we present the reasoned statement of the Italian Society of Hypertension to maintain ongoing antihypertensive treatments. Furthermore, the Italian Society of Hypertension presents its own initiative to investigate the issue using an online questionnaire to collect relevant data in human disease.
\end{abstract}

Keywords COVID-19 $\cdot$ hypertension · cardiovascular diseases · infection · outcomes

\section{Introduction}

The recent Severe Acute Respiratory Syndrome (SARS) due to Coronavirus 2 (CoV-2) infection pandemic and subsequent spread of the disease called COVID-19 brought back to discussion a topic already highlighted during the SARSCoV-1 and Coronavirus-related SARS known as the Middle East Respiratory Distress Syndrome (MERS) of 2002 and 2013. During those outbreaks it was observed a particularly elevated incidence of cardiovascular disease among patients, who were also characterized by being elderly and, in particular during the MERS, prevalently male $[1,2]$.

Another main topic of the discussion is the role of the angiotensin-converting enzyme (ACE) 2. Indeed, this carboxypeptidase has been identified as a functional receptor for the spike protein of the coronaviruses' outer membrane,

Guido Grassi

guido.grassi@unimib.it

Extended author information available on the last page of the article including SARS-CoV-2 [3]. ACE2 is strongly expressed in the epithelium of different organs, such as the kidney, heart, and lungs.

ACE2 shares a large affinity to the amino peptidase ACE that is target to the ACE inhibitors, a class of antihypertensive drugs. ACE inhibitors represent the most used class of cardiovascular agents in the world, for the treatment of epidemic cardiovascular conditions such as hypertension and heart failure. Although not directly inhibited by ACE inhibitors, ACE2 is affected by chronic treatment with this class of drugs, which leads to an increase in ACE2 expression in several tissues [4]. Interestingly, this feature is also shared by another class of drugs, the angiotensin receptor-1 blockers (ARBs), whose chronic administration is as well able to increase the level of expression of ACE2 and also its activity, as assessed by the circulating levels of the ACE2 product, angiotensin 1-7 [5]. These findings support the concern that the treatment with Renin-Angiotensin System (RAS) inhibitors could make COVID-19 symptoms more severe due to increased expression of ACE2. 


\section{Cardiovascular Disease and COVID-19}

Remarkably, both issues have been re-proposed in the occurrence of the present epidemic of COVID-19. In particular, the issue of the prevalence of cardiovascular diseases among COVID-19 patients is proposed by observational data obtained in Chinese [6] and Italian patients [7].

In this context, we still lack the analysis of the confounding effects of age on the apparent association between cardiovascular disease COVID-19 infection and clinical severity. Indeed, the observed prevalence of male and elderly patients, observed especially in the Italian COVID-19 population, is a confounding factor that needs to be corrected for before any conclusive association is drawn. This concern has been expressed by many [8-10].

Similarly, the ACE2 upregulation argument has never been demonstrated in humans. Indeed, while there is conflicting evidence from animal studies that ARBs (probably not ACE inhibitors) may upregulate membrane-bound ACE2 in tissue-specific manners (e.g., heart but not kidney), these data cannot be extrapolated to humans, and are not sufficient to support facilitation of SARS-CoV-2 entry [9]. In particular, it has never been demonstrated that the ACE2 upregulation in the human lung occurs upon RAS inhibition, and even less that this causes a worsening of the COVID-19 disease. Furthermore, it can also be speculated that ACE2 upregulation is protective. Indeed, it has been shown that the binding of coronavirus to ACE2 leads to the downregulation of ACE2 [11], which in turn causes an ACE/ACE2 imbalance and to the excessive production of angiotensin II by the related ACE enzyme. This excess of Angiotensin II stimulates angiotensin II receptor type 1 (AT1R) and might cause an increase in pulmonary vascular permeability and lung damage [12]. Therefore, according to this hypothesis, the upregulation of ACE2, caused by the chronic intake of AT1R and ACE Inhibitors, could be protective through two mechanisms: first, by blocking the increased production of angiotensin $1-7$, which has been advocated as a possible mechanism of protection for the lung; second, by reducing the production of Angiotensin II, it removes a cause of lung damage [13].

\section{Recommendations}

In this context of uncertainty, there are advocates within the scientific community raising their voices for the cessation of ACE inhibitors and ARBs among patients taking these drugs, that is claimed for both the prevention of the infection and the attenuation of the symptoms in case of infection. These speculative claims are then taken over by the laical press, starting a word of mouth that sustains panic behaviors among the general population.

The Italian Society of Hypertension (SIIA) therefore takes a clear and firm position, in line with statements by other International Societies (See Table 1). SIIA states the following items:

1. There is no evidence to associate hypertension or other CVDs with COVID-19 disease: if hypertension was a predisposing factor for coronavirus infection, there should be more hypertensives among COVID-19 patients than observed in the general population; to date, there is no evidence that people with hypertension are overrepresented among those infected with COVID-19.

2. There is no clinical evidence in humans that associates the intake of ACE inhibitors or ARBs with COVID-19 disease. At present we can neither say that they improve nor say they worsen the susceptibility to coronavirus infection.

3. There are no clinical data in patients that can confirm the harmful effect (not even the protective one) of ACE inhibitors and ARB in the context of the COVID19 epidemic.

Furthermore, SIIA reiterates that subsequent points:

A. Acute suspension without medical control of cardiovascular therapy and specifically of antihypertensive treatment increases the occurrence of acute events, including hypertensive emergencies, heart failure decompensation, heart attacks, and stroke.

B. The favorable effects of ACE inhibitors and ARBs on the control of the progression of CVDs in general and in particular of hypertensive patients have been confirmed for many years and for this reason RAS inhibitors represent central agents for the management of cardiovascular conditions that cannot be easily substituted [10].

C. In hypertensive patients with COVID-19 or at risk of COVID-19 infection, ACE inhibitors and ARBs treatment should be maintained according to the recommendations contained in the $2018 \mathrm{ESC} / \mathrm{ESH}$ guidelines [10].

D. Similarly, in all patients currently on therapy with ACE inhibitors, ARBs and in the case of patients with heart failure, also the ARNIs, these drugs must not be suspended.

E. In patients with COVID-19 with severe symptoms or sepsis, ACE Inhibitors and ARBs, like all other antihypertensive drugs, should be used or discontinued on a case by case basis, taking into account current guidelines. 


\section{Further Scientific Research}

SIIA hopes and promotes further research that analyzes the constantly increasing data on the impact of hypertension and antihypertensive drugs, in particular ACE inhibitors and ARBs, on the clinical course of COVID-19 infections, in order to update these positions as new evidence becomes available.

In particular, to clarify which mechanism is prevalent and has a role in the clinical manifestation of COVID-19, SIIA has launched a fact-finding investigation to verify the impact of therapy with inhibitors of the renin-angiotensin system on the onset and clinical manifestation of the disease COVID19. This observational research is based on an online questionnaire made of 18 questions to collect information on medical history and the evolution of the disease in COVID19 patients (ID: NCT04331574; clinicaltrials.gov).

\section{Compliance with Ethical Standards}

Conflict of Interest On behalf of all authors, the Corresponding Author states that there is no conflict of interest.

Open Access This article is licensed under a Creative Commons Attribution-NonCommercial 4.0 International License, which permits any non-commercial use, sharing, adaptation, distribution and reproduction in any medium or format, as long as you give appropriate credit to the original author(s) and the source, provide a link to the Creative Commons licence, and indicate if changes were made. The images or other third party material in this article are included in the article's Creative Commons licence, unless indicated otherwise in a credit line to the material. If material is not included in the article's Creative Commons licence and your intended use is not permitted by statutory regulation or exceeds the permitted use, you will need to obtain permission directly from the copyright holder.To view a copy of this licence, visit http://creativecommons.org/licenses/by-nc/4.0/.

\section{References}

1. Assiri A, Al-Tawfiq JA, Al-Rabeeah AA, Al-Rabiah FA, Al-Hajjar $\mathrm{S}, \mathrm{Al}-\mathrm{Barrak} \mathrm{A}$, et al. Epidemiological, demographic, and clinical characteristics of 47 cases of Middle East respiratory syndrome coronavirus disease from Saudi Arabia: a descriptive study. Lancet Infect Dis. 2013;13(9):752-61. doi:https://doi.org/10.1016/ S1473-3099(13)70204-4.

2. Leung GM, Hedley AJ, Ho LM, Chau P, Wong IO, Thach TQ, et al. The epidemiology of severe acute respiratory syndrome in the 2003 Hong Kong epidemic: an analysis of all 1755 patients. Ann Intern Med. 2004;141(9):662-73. doi:https://doi. org/10.7326/0003-4819-141-9-200411020-00006.

3. Menachery VD, Yount BL Jr, Debbink K, Agnihothram S, Gralinski LE, Plante JA, et al. A SARS-like cluster of circulating bat coronaviruses shows potential for human emergence. Nat Med. 2015;21(12):1508-13. doi:https://doi.org/10.1038/nm.3985.

4. Zisman LS, Meixell GE, Bristow MR, Canver CC. Angiotensin-(1-7) formation in the intact human heart: in vivo dependence 
on angiotensin II as substrate. Circulation. 2003;108(14):1679-81. doi:https://doi.org/10.1161/01.CIR.0000094733.61689.D4.

5. Ferrario CM, Jessup J, Chappell MC, Averill DB, Brosnihan $\mathrm{KB}$, Tallant EA, et al. Effect of angiotensin-converting enzyme inhibition and angiotensin II receptor blockers on cardiac angiotensin-converting enzyme 2. Circulation. 2005;111(20):2605-10. doi:https://doi.org/10.1161/CIRCULATIONAHA.104.510461.

6. Chen N, Zhou M, Dong X, Qu J, Gong F, Han Y, et al. Epidemiological and clinical characteristics of 99 cases of 2019 novel coronavirus pneumonia in Wuhan, China: a descriptive study. Lancet. 2020;395(10223):507-13. doi:https://doi.org/10.1016/ S0140-6736(20)30211-7.

7. Onder G, Rezza G, Brusaferro S. Case-Fatality Rate and Characteristics of Patients Dying in Relation to COVID-19 in Italy. JAMA. 2020. doi:https://doi.org/10.1001/jama.2020.4683.

8. Clerkin KJ, Fried JA, Raikhelkar J, Sayer G, Griffin JM, Masoumi A, Disease Coronavirus, et al. (COVID-19) and cardiovascular disease. Circulation. 2019. https://doi.org/10.1161/CIRCULATIO NAHA.120.046941.
9. Danser AHJ, Epstein M, Batlle D. Renin-angiotensin system blockers and the COVID-19 pandemic. Hypertension. 2020. https ://doi.org/10.1161/HYPERTENSIONAHA.120.15082.

10. Hanff TC, Harhay MO, Brown TS, Cohen JB, Mohareb AM. Is There an Association Between COVID-19 Mortality and the ReninAngiotensin System-a Call for Epidemiologic Investigations. Clin Infect Dis. 2020. doi:https://doi.org/10.1093/cid/ciaa329.

11. Glowacka I, Bertram S, Herzog P, Pfefferle S, Steffen I, Muench MO, et al. Differential downregulation of ACE2 by the spike proteins of severe acute respiratory syndrome coronavirus and human coronavirus NL63. J Virol. 2010;84(2):1198-205. doi:https://doi. org/10.1128/JVI.01248-09.

12. Imai $Y$, Kuba K, Rao S, Huan Y, Guo F, Guan B, et al. Angiotensin-converting enzyme 2 protects from severe acute lung failure. Nature. 2005;436(7047):112-6. doi:https://doi.org/10.1038/natur e03712.

13. Gurwitz D. Angiotensin receptor blockers as tentative SARSCoV-2 therapeutics. Drug Dev Res. 2020. doi:https://doi. org/10.1002/ddr.21656.

\section{Affiliations}

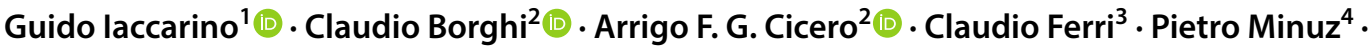

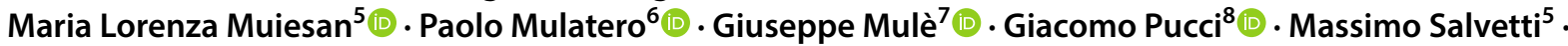

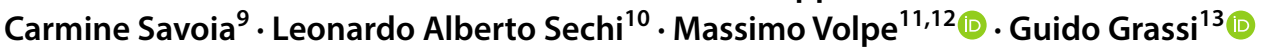

Guido Iaccarino

guiaccar@unina.it

Claudio Borghi

claudio.borghi@unibo.it

Arrigo F. G. Cicero

arrrigo.cicero@unibo.it

Claudio Ferri

claudio.ferri@cc.univaq.it

Pietro Minuz

pietro.minuz@univr.it

Maria Lorenza Muiesan

marialorenza.muiesan@unibs.it

Paolo Mulatero

paolo.mulatero@unito.it

Giuseppe Mulè

giuseppe.mule@unipa.it

Giacomo Pucci

giacomo.pucci@gmail.com

Massimo Salvetti

massimo.salvetti@unibs.it

Carmine Savoia

carmine.savoia@uniroma1.it

Leonardo Alberto Sechi

sechi@uniud.it

Massimo Volpe

massimo.volpe@uniroma1.it

1 Department of Advanced Biomedical Sciences, Federico II University, Naples, Italy
Department of Medicine and Surgery Sciences, Alma Mater Studiorum University of Bologna, Bologna, Italy

3 Department of Clinical Medicine, Public Health, Life and Environment Sciences, University of Aquila, L'Aquila, Italy

4 Department of Medicine, University of Verona, Verona, Italy

5 Dept of Clinical \& Experimental Sciences, University of Brescia-Medicina 2, ASST Spedali Civili Brescia, Brescia, Italy

6 Division of Internal Medicine and Hypertension, Department of Medical Sciences, University of Torino, Turin, Italy

7 Department of Health Promotion, Mother and Child Care, Internal Medicine and Medical Specialties, Unit of Nephrology and Hypertension, University of Palermo, Palermo, Italy

8 Section of Internal Medicine Terni, Department of Medicine, University of Perugia, Terni, Italy

9 Clinical and Molecular Medicine Department, Sapienza University of Rome, Rome, Italy

10 Department of Medical Area, University of Udine, Udine, Italy

11 Division of Cardiology, Department of Clinical and Molecular Medicine, University of Rome Sapienza, Sant'Andrea Hospital, Rome, Italy

12 IRCCS Neuromed, Pozzilli, IS, Italy

13 Department of Medicine and Surgery, University of Milano-Bicocca, Milan, Italy 